\title{
Comment je prends en charge la nutrition d'un patient en état de choc
}

\section{Nutritional Support in Critically Ill Patients}

\author{
F. Tamion · J. Bohé \\ Reçu le 3 juillet 2018; accepté le 30 septembre 2018 \\ (C) SRLF et Lavoisier SAS 2018
}

Résumé La réponse métabolique à l'agression correspond à un ensemble de réactions à la base de l'adaptation de l'organisme aux nouvelles conditions. Ces modifications concernent des aspects métaboliques spécifiques comme le maintien de la masse protéique et/ou l'état des réserves énergétiques. L'une des principales difficultés de l'optimisation du support métabolique consiste à distinguer les changements métaboliques bénéfiques de ceux qui sont délétères pour l'organisme. Dans ce contexte, les objectifs thérapeutiques peuvent se limiter à une approche nutritionnelle s'attachant à limiter le déficit énergétique et les pertes protéiques et musculaires. Ils peuvent être plus ambitieux en essayant d'adapter les apports aux différents besoins d'un point de vue quantitatif comme qualitatif. La limitation du déficit énergétique semble être un objectif raisonnable à atteindre selon les données de la littérature. Enfin, essayer d'interférer avec la réponse métabolique à l'agression (immunomodulation, manipulations pharmacologiques des voies métaboliques, etc.) représente le degré d'intervention métabolique le plus élaboré et, si quelques données ont pu être encourageantes, il n'est pas possible d'affirmer que cet objectif soit complètement réaliste, voire même bénéfique. Les apports nutritionnels doivent être intégrés à la stratégie thérapeutique globale de prise en charge. La réponse optimale du support nutritionnel a pour but « de donner les moyens métaboliques » de la guérison.

Mots clés Support nutritionnel · État de choc . Métabolisme
Abstract The metabolic response to aggression corres- ponds to different reactions that form the basis of the body's
F. Tamion $(\bowtie)$
Service de réanimation médicale, U1096, UNIROUEN, Normandie Université, CHU de Rouen, 1, rue de Germont, F-76031 Rouen cedex, France
e-mail : fabienne.tamion@chu-rouen.fr
J. Bohé
Unité de réanimation médicale, pôle des urgences, centre hospitalier Lyon-Sud, F-69310 Pierre-Bénite, France

adaptation to new context. These modifications concern specific metabolic aspects such as the maintenance of protein mass and/or the state of energy reserves. One of the main difficulties in optimizing metabolic support is to distinguish beneficial metabolic changes from those that are harmful to the body. In this context, therapeutic ambitions can be limited to a nutritional approach focusing on limiting the energy deficit, protein, and muscle losses. They can be more ambitious by trying to adapt the different needs from both a quantitative and qualitative point of view. According to the literature, limiting the energy deficit seems to be a major objective to achieve. Finally, trying to interfere with the metabolic response to aggression (immuno-modulation, pharmacological manipulations of metabolic pathways, etc.) represents the most elaborate degree of metabolic intervention but it is not possible to say that this objective is completely realistic, or even beneficial. Nutritional intakes should be integrated into the overall therapeutic management strategy. The optimal nutritional support response aims to "provide the metabolic means" for healing.

Keywords Nutritional support $\cdot$ Shock $\cdot$ Metabolism

\section{Rappels physiologiques}

Dans les situations aiguës telles qu'on les observe en réanimation, le changement métabolique peut être un profond bouleversement et correspondre à une véritable redistribution des priorités permettant à l'organisme une adaptation aux nouvelles conditions. L'une des principales difficultés de l'optimisation des apports nutritionnels consiste à distinguer les changements métaboliques bénéfiques qui doivent être conservés de ceux qui sont délétères. La question est d'autant plus complexe que selon l'intensité, la durée ou le contexte clinique une même modification peut être successivement bénéfique ou délétère. Dans ce contexte, les objectifs thérapeutiques peuvent se limiter à une approche nutritionnelle simple en s'attachant seulement à couvrir a minima les 
besoins énergétiques et à limiter les pertes protéiques. Ils peuvent être plus ambitieux en essayant d'adapter le plus exactement possible les apports aux différents besoins, d'un point de vue quantitatif comme qualitatif. Enfin, essayer d'interférer avec la réponse métabolique à l'agression (immunomodulation, manipulations pharmacologiques des voies métaboliques, de la synthèse protéique, etc.) représente le degré d'intervention métabolique le plus élaboré, et si les données physiopathologiques ont été encourageantes, l'impact clinique reste à démontrer. Il est important de garder à l'esprit que la réponse à l'agression a pour but « de donner les moyens métaboliques » et non de maintenir «normales » de grandes constantes dites physiologiques, caractéristiques d'un organisme en bonne santé. Dans ces situations d'agres- sion sévère comme l'état de choc, le support nutritionnel doit être au service de la stratégie thérapeutique globale de prise en charge. Des apports trop élevés chez des patients en état de choc réfractaire peuvent être délétères, alors qu'il sera important de prévenir la dénutrition protéinoénergétique pour permettre la réhabilitation (Fig. 1).

\section{Modification métabolique au cours de l'agression}

La réponse métabolique à l'agression s'oppose totalement au jeûne : non seulement il n'y a pas de réduction des dépenses énergétiques ni du catabolisme protéique, mais on observe au contraire une importante augmentation de ces deux paramètres. Il n'y a pas de réduction de l'insulinémie, mais une
A

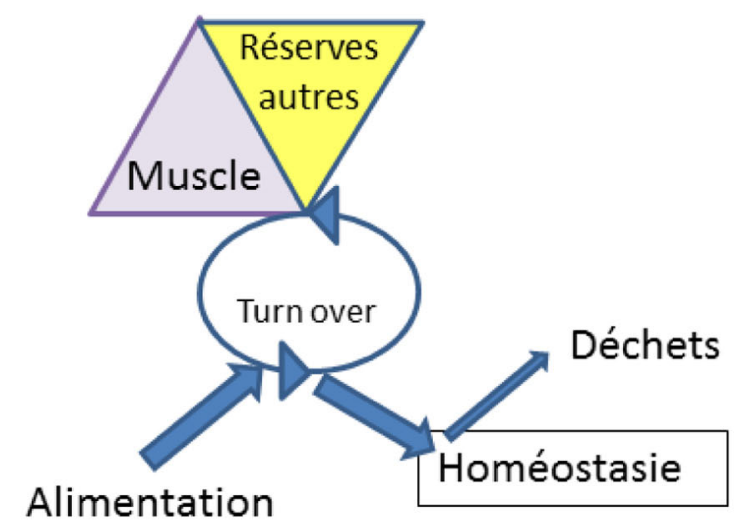

C

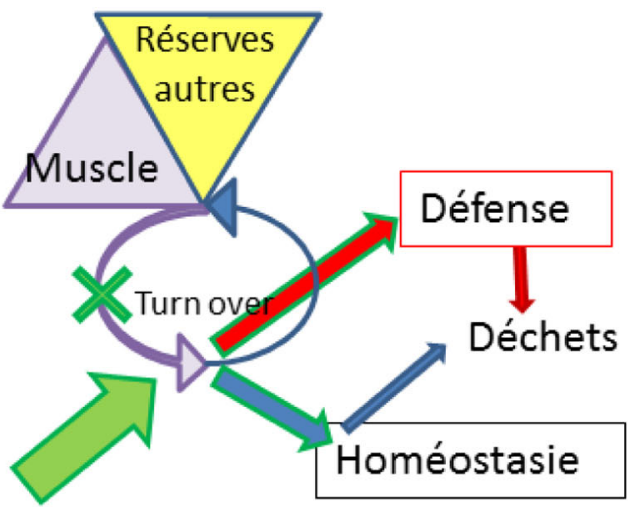

\section{NUTRITION}

B

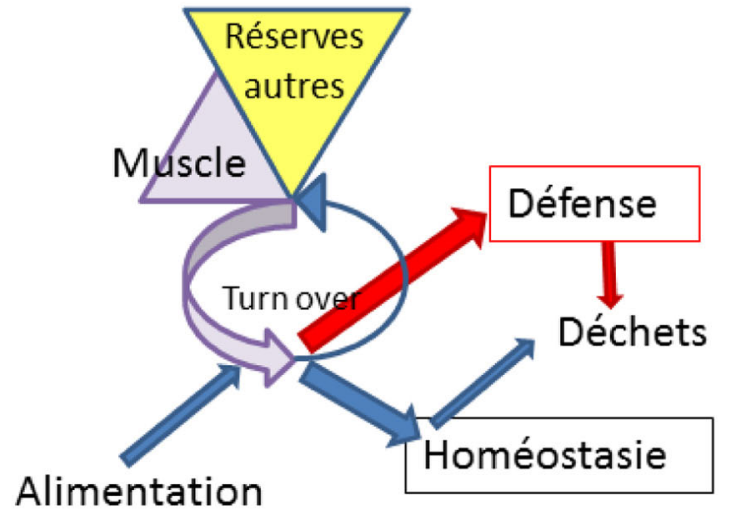

D

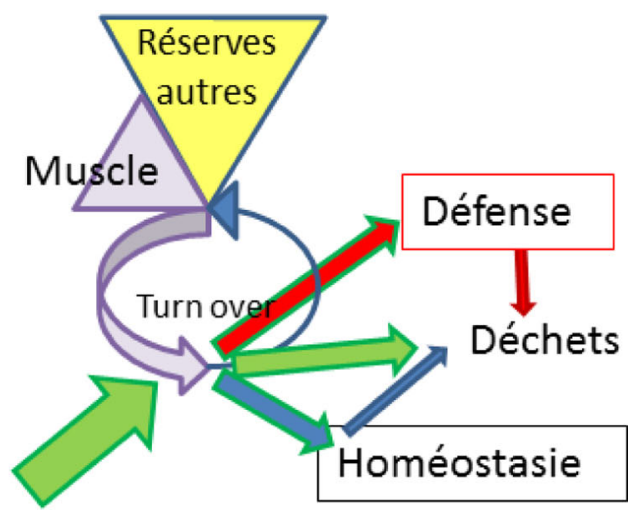

NUTRITION

Fig. 1 Place de la nutrition artificielle au cours de l'agression. A. Chez l'adulte en situation normale, non agressé, les prises alimentaires permettent d'assurer l'homéostasie. B. Au cours d'une agression, les prises alimentaires sont réduites alors que les besoins énergétiques et en protéines sont augmentés pour permettre la défense de l'organisme. L'énergie et les acides aminés sont alors prélevés dans les réserves endogènes et principalement le muscle. C. L'objectif de la nutrition artificielle est de couvrir les besoins énergétiques et protéiques accrus qui ne sont pas couverts par les prises alimentaires, en protégeant les réserves de l'organisme. D. Une nutrition artificielle peut être délétère (surnutrition) si elle ne limite pas l'utilisation des réserves endogènes. 
augmentation, parallèlement à un état d'insulinorésistance, en rapport avec l'élévation des hormones dites de contrerégulation (glucagon, cortisol et catécholamines) [1]. En fait, si la réponse générale à l'agression est opposée à l'adaptation au jeûne, les différentes voies métaboliques ne sont pas impliquées de manière similaire. Ainsi, la production hépatique et le renouvellement du glucose (turnover) sont augmentés, alors que son oxydation relative (par rapport au niveau de la glycémie) est diminuée. L'absence de cétogenèse ne permet pas de réduire le catabolisme protéique responsable de la perte de masse musculaire au cours des états d'agression. La réponse métabolique à l'agression est caractérisée par une accélération importante du métabolisme, en particulier protéique, allant dans le sens du catabolisme aux dépens de l'anabolisme.

\section{Modifications des grandes voies métaboliques : les hydrates de carbone}

L'agression perturbe largement le métabolisme du glucose, contribuant au développement d'une hyperglycémie chez les patients de réanimation, en rapport avec une insulinorésistance. Si la glycémie des patients traumatisés est comparable à celle des volontaires, cela est obtenu au prix d'une concentration plasmatique d'insuline plus importante, ce qui témoigne de l'insulinorésistance [2]. Ces patients ont une production accrue de glucose par activation de la gluconéogenèse hépatique à partir de précurseurs tels que le lactate, le glycérol, mais surtout les acides aminés. La glycogénogenèse est également diminuée sous l'effet des hormones de stress [3]. L'élévation des concentrations circulantes du glucagon, du cortisol et des catécholamines explique l'augmentation du turnover du glucose.

Les cytokines stimulent la captation du glucose, principalement au niveau des cellules immunes (macrophages, rate, foie, poumons), sous la dépendance du transporteur de type GLUT1. Par ailleurs, l'activité des différents récepteurs insuliniques est diminuée de près de $50 \%$, ce qui conduit parallèlement à une altération de l'entrée de glucose dans les tissus dits insulinodépendants (muscle, foie, tissus adipeux) [4]. L'inhibition de l'action de l'insuline au niveau de ces tissus, grands consommateurs de glucose, proviendrait d'un effet inhibiteur de certains médiateurs de l'inflammation sur la voie intracellulaire de l'insuline et d'une diminution de la translocation des transporteurs de glucose de type GLUT4. Ainsi, il a été démontré que les cytokines pro-inflammatoires inhibent la cascade de phosphorylation au niveau de la protéine kinase B (PTP1B), indispensable à l'action de l'insuline.

Parallèlement, l'oxydation du glucose est altérée [5]. En fait, il s'agit d'une modification relative, c'est-à-dire que l'augmentation de l'oxydation du glucose est faible (voire absente), alors que l'augmentation du métabolisme global du glucose est conséquente [6]. Cela indique que l'inflammation est responsable d'une augmentation importante du renouvellement du glucose qui ne porte pas sur son métabolisme oxydatif, mais sur la part non oxydative.

\section{Modifications des grandes voies métaboliques : les lipides}

L'agression va s'accompagner d'une augmentation de la dépense énergétique. En retour, les triglycérides endogènes stockés dans le tissu adipeux et les triglycérides exogènes sont hydrolysés afin de libérer des acides gras et du glycérol. La majorité des travaux retrouve une augmentation de l'oxydation lipidique qui coexiste avec une réduction de l'activité de la lipoprotéine lipase endothéliale, l'ensemble étant responsable d'une élévation des triglycérides plasmatiques [7]. La lipolyse accrue a pour conséquence une augmentation des concentrations plasmatiques en acides gras libres et en glycérol. En fait, la production endogène d'acides gras libres excède le plus souvent leur utilisation. Du fait de la variété des atteintes organiques potentielles (fonction hépatique, insulinorésistance, hypoalbuminémie, hypoperfusion, hypoxie), on peut rencontrer soit une élévation importante des triglycérides, soit, au contraire, des valeurs faibles dans le cas d'une déchéance hépatique ne permettant plus leur synthèse [8]. Le glycérol libéré sera transformé en glucose au niveau hépatique grâce à la néoglucogenèse. Les acides gras sont en partie réestérifiés en triglycérides libérés dans la circulation sous forme de lipoprotéines de type VLDL (very low density lipoprotein) pour atteindre les tissus périphériques. Tous ces intermédiaires métaboliques sont utilisés comme substrats énergétiques dans différents tissus.

\section{Modifications des grandes voies métaboliques : les protéines}

L'hypercatabolisme protéique au cours de l'agression a été très anciennement mis en évidence par l'augmentation des pertes d'azote dans les urines. L'origine musculaire de cet azote dans le contexte de la réponse à l'agression démontre bien la redistribution du matériel protéique du territoire musculaire [9] vers d'autres organes comme le foie ou les tissus agressés. Ce phénomène est sous la dépendance des modifications endocrines et du processus inflammatoire. L'augmentation du turnover protéique s'accompagne d'une augmentation du catabolisme des acides aminés, comme en témoigne l'augmentation des pertes azotées urinaires, proportionnelle à l'intensité de l'agression. Ainsi, le bilan protéique net est négatif avec une perte protéique corporelle, notamment au niveau musculaire. Ainsi, les patients peuvent perdre 10 à $25 \%$ de la teneur en protéines de leur corps, soit une perte pouvant aller jusqu'à $1-3 \mathrm{~kg}$ de perte musculaire par semaine sur les dix premiers jours. De nombreuses 
études ont montré une relation pronostique entre la morbimortalité et la perte de masse maigre des patients à l'admission. Dans ces travaux, il est bien démontré que non seulement la quantité, mais aussi la qualité de masse maigre sont des éléments majeurs du pronostic [10]. Les modifications des concentrations plasmatiques des acides aminés ont fait l'objet de nombreux travaux. Comme dans le cas du jeûne, il existe une diminution de la concentration plasmatique des acides aminés non essentiels, tandis que les essentiels sont, dans l'ensemble, peu modifiés [11]. Mais, à l'opposé de la situation de jeûne où le catabolisme protéique est réduit, la coexistence d'un hypercatabolisme avec une réduction des concentrations circulantes des acides aminés montre bien l'avidité avec laquelle ils sont métabolisés au cours de l'agression. La concentration plasmatique de la glutamine est le plus souvent réduite ; ce point semble important, en particulier au niveau du métabolisme de l'entérocyte, des cellules immunologiquement compétentes et peut-être aussi dans la régulation de la synthèse protéique en général [12]. Les acides aminés ramifiés, valine, leucine et isoleucine, sont catabolisés au niveau des muscles squelettiques, leur concentration plasmatique est peu modifiée en règle générale par l'agression [13].

En résumé, les agressions sévères s'accompagnent d'un ensemble de phénomènes métaboliques dont la durée et l'intensité dépendent de la sévérité du stress, pouvant aboutir à une dénutrition très rapide. Différents phénomènes neurohormonaux et inflammatoires vont se succéder et déterminer les changements suivants :

- une augmentation de la dépense énergétique ;

- un état d'insulinorésistance, résultat d'une élévation simultanée de l'insuline et des hormones dites contrerégulatrices (glucagon, cortisol, catécholamines) ;

- une augmentation de l'oxydation des lipides qui coexiste avec une réduction de l'activité de la lipoprotéine lipase adipocytaire. La conséquence de ces deux modifications est une élévation des triglycérides plasmatiques ;

- un catabolisme protéique intense, non compensé par l'augmentation des synthèses protéiques conduisant à une perte de masse maigre impliquée dans l'altération de la fonction musculaire.

Ainsi, l'agression va rapidement dégrader l'état nutritionnel et être à l'origine de conséquences délétères multiples en termes de morbimortalité, soutenant la nécessité d'une prise en charge métabolique pour tout patient agressé. Ainsi, le monitorage de cette réponse métabolique va être un enjeu majeur des prochaines années, puisqu'il n'existe aucun marqueur clinique ou biologique disponible actuellement. C'est dans cette recherche que le profil «métabolomique »a été rapporté comme une approche prometteuse pour caractériser les changements métaboliques au cours de l'agression [14].

\section{Besoins caloriques et protéiques chez le patient en état de choc}

\section{Évaluation de la dénutrition}

Tout patient admis en réanimation pour une durée présumée supérieure à trois jours est à risque de dénutrition. Le dépistage de la dénutrition représente l'étape initiale essentielle dans la prise en charge du risque métabolique : tout patient hospitalisé en réanimation devrait donc bénéficier rapidement après son admission d'une évaluation nutritionnelle incluant la détermination de son risque nutritionnel, de ses besoins protéinoénergétiques et de ses apports protéinoénergétiques. Cette évaluation permet de déterminer précocement la stratégie de prise en charge nutritionnelle la mieux adaptée au patient.

Compte tenu du faible nombre d'études, des résultats discordants et de la faiblesse du niveau de preuve, le recueil de l'IMC à l'admission semble pouvoir être recommandé, de même que la recherche d'une perte de poids récente.

\section{Évaluation de la dépense énergétique}

En cas d'agression sévère, la dépense énergétique peut augmenter de 10 à $100 \%$ d'un individu à un autre en fonction de paramètres intrinsèques ou extrinsèques [15]. La fièvre, l'agitation, la douleur, la toux sont, entre autres, des facteurs augmentant la dépense énergétique. Certaines situations pathologiques sont clairement hypermétaboliques. Chez les patients cérébrolésés, la dépense énergétique peut augmenter de 20 à $30 \%$; chez le polytraumatisé sans atteinte cérébrale, cette augmentation sera de 20 à $50 \%$. Les grands brûlés ont une augmentation de la dépense énergétique de 25 à $80 \%$ selon les études et l'étendue des lésions. Il est cependant difficile de faire une corrélation statistiquement satisfaisante entre les indices de gravité utilisés en réanimation et l'augmentation de la dépense énergétique à l'échelle individuelle.

De nombreuses thérapeutiques influencent positivement ou négativement la dépense énergétique. Ainsi, la sédation profonde utilisée chez les neurotraumatisés ou en cas de syndrome de détresse respiratoire aiguë (SDRA) pourrait réduire la dépense énergétique de repos (DER) de plus de $25 \%$. Les facteurs de correction utilisés pour les équations de type Harris-Benedict découlent de ces déterminants de variation de la DER. Ils sont probablement peu fiables, étant donné la complexité de ces situations. De plus, la dépense énergétique évolue dans le temps. En effet, l'équipe de Plank et Hill a montré que la dépense énergétique évolue au cours du temps chez les patients admis en réanimation pour traumatismes multiples ou en postopératoire de péritonite [16]. Elle se situait aux environs de 20 à $25 \mathrm{kcal} / \mathrm{kg}$ par jour de $\mathrm{j} 3$ à $\mathrm{j} 5$, puis augmentait jusqu'au dixième jour pour atteindre 
$30 \mathrm{kcal} / \mathrm{kg}$ par jour. Enfin, en phase de réhabilitation postagressive, la dépense énergétique est évidemment considérablement augmentée et se situe probablement au-delà de $40 \mathrm{kcal} / \mathrm{kg}$, mais est extrêmement variable d'un patient à l'autre.

La calorimétrie indirecte est considérée comme la mesure de référence dans l'évaluation de la dépense énergétique [17] bien qu'il soit reconnu que les preuves sur lesquelles se fonde cette recommandation sont limitées en réanimation. La vraie question est de savoir si les calories fournies aux patients pendant la phase aiguë de l'état de choc devraient correspondre aux mesures estimées par la calorimétrie, qui ne prennent pas en compte la production endogène de métabolites énergétiques et ainsi le risque de surnutrition (Fig. 1).

\section{Quel apport calorique ?}

Les dernières recommandations de la SFAR-SRLF-SFNEP [18] indiquent que :

- pour évaluer précisément la dépense énergétique d'un patient de réanimation, il faut utiliser la calorimétrie indirecte (méthode de référence en tenant compte de ses limites d'utilisation) plutôt que les équations prédictives (accord faible) ;

- en cas d'utilisation de calorimétrie indirecte, il ne faut probablement pas dépasser la dépense énergétique mesurée (accord faible) ;

- en l'absence de calorimétrie indirecte, il faut probablement avoir un objectif calorique total de $20-25 \mathrm{kcal} / \mathrm{kg}$ par jour à la phase aiguë et de $25-30 \mathrm{kcal} / \mathrm{kg}$ par jour après stabilisation (accord faible) ;

- il est important de prendre en compte l'ensemble des apports caloriques, notamment de comptabiliser les apports engendrés par l'administration de glucosé ou de propofol ;

- il faut probablement majorer l'apport protéique quotidien du patient sous épuration extrarénale continue pour atteindre un niveau de 1,7 à $2 \mathrm{~g} / \mathrm{kg}$ par jour. En effet, les pertes en acides aminés ou en protéines varient essentiellement en fonction de leur concentration plasmatique et dans une moindre mesure de la technique d'épuration extrarénale continue choisie (10 à $17 \%$ ).

On considère dans ces recommandations que les besoins caloriques, et donc les apports recommandés, correspondent à la dépense énergétique de base ou de repos. Mais cela peut être discuté, car la surnutrition est au moins aussi dangereuse que la sous-nutrition.

Une diminution significative de la mortalité a été observée lorsque l'apport calorique couvre $70 \%$ des besoins. Il existe une corrélation positive entre l'augmentation du déficit énergétique au cours du séjour en réanimation et la fréquence de complications telles que les infections liées aux soins, le syndrome de détresse respiratoire de l'adulte, l'insuffisance rénale, le recours à la chirurgie et la survenue d'escarres $[19,20]$. Cela est d'autant plus vrai que le patient présentait une dénutrition à l'admission en réanimation [21]. Une augmentation de la mortalité, de la durée de séjour et du taux d'infection a été observée lorsque l'apport calorique était supérieur à $70 \%$ des besoins à la phase initiale. Il est primordial d'éviter la surnutrition et ses complications métaboliques : hyperglycémie, hépatopathie dont la stéatose hépatique, associées à une augmentation de la morbimortalité. Cela est soutenu par l'échec de certaines études d'optimisation des apports caloriques [22].

Les objectifs caloriques et protéiques optimaux restent flous, de même que le moment optimal du début de la mise en place du support nutritionnel. Un essai comparant la sousnutrition permissive (40-60\% de la cible calorique) avec un apport standard (70-100\% de la cible calorique) et avec un apport en protéines similaire dans les deux bras n'a trouvé aucune différence significative de mortalité à j90 en réanimation et hospitalière ni en termes d'intolérance alimentaire, de diarrhée ou de taux d'infection acquise [23]. Un autre essai comparant un apport hypocalorique $(15 \mathrm{kcal} / \mathrm{kg}$ par jour) et les recommandations ( $25 \mathrm{kcal} / \mathrm{kg}$ par jour) avec un apport protéique similaire $(1,7 \mathrm{~g} / \mathrm{kg}$ par jour) n'a montré aucune différence significative dans la mortalité à j28, la durée de séjour, la durée de la ventilation mécanique ou les scores SOFA [24]. Enfin, une étude comparant un apport hypocalorique versus normocalorique a observé plus d'infections nosocomiales dans le groupe hypocalorique $(26,1$ versus $11,1 \%$ ) [25]. Dans cet essai, l'apport en protéines était également significativement plus faible dans le groupe hypocalorique. Ces différents résultats peuvent suggérer que le support nutritionnel hypocalorique à la phase aiguë de l'agression n'est pas délétère tant qu'un apport adéquat en protéines est observé. Deux méta-analyses comparant ces deux approches à la phase aiguë ne montrent aucune différence dans la mortalité globale, le taux de complications infectieuses, l'intolérance gastro-intestinale ou la durée de la ventilation mécanique [26,27]. La plupart des patients inclus étaient non dénutris (IMC élevé, faible score NutriScore), et l'extrapolation de ces données aux patients avec un risque nutritionnel élevé ne peut être considérée.

Enfin, des études récentes ont montré que des apports entéraux, dits trophiques, de l'ordre de $300 \mathrm{kcal}$ par jour pendant les cinq premiers jours de réanimation (versus $1400 \mathrm{kcal}$ par jour dans le groupe témoin), n'induisaient ni surmorbidité ni surmortalité [28].

L'urgence est donc à ne pas surnourrir les patients à la phase aiguë sans induire de dénutrition. Il apparaît clairement qu'il manque à la fois d'outils pour définir les objectifs énergétiques de ces patients agressés et d'études pour définir l'apport optimal à cette phase de l'agression. 


\section{Quel apport protéique?}

Les dernières recommandations de la SFAR-SRLF-SFNEP [18] indiquent qu'il faut apporter entre 1,2 et $2,0 \mathrm{~g} / \mathrm{kg}$ par jour de protéines.

Les données concernant les besoins protéiques en réanimation sont beaucoup plus rares. L'étude la plus pertinente a été réalisée en 1998 chez 23 patients de réanimation ( 15 patients polytraumatisés et 5 traités pour sepsis) [29]. L'évolution de la masse protéique totale, mesurée par DEXA, était moins défavorable chez les patients recevant $1,47 \mathrm{~g}$ de protéines par kilogramme de masse maigre et par jour, en comparaison à ceux recevant 1,14 ou $1,86 \mathrm{~g}$. Dans l'étude de Koretz et al., la balance de l'azote était moins négative chez les patients recevant $1,5 \mathrm{~g}$ de protéines par kilogramme de poids total et par jour [30]. Des études observationnelles montrent qu'un apport majoré en protéines est associé à une meilleure survie. Mehta et al. ont montré que l'apport en protéines est linéairement associé à une diminution de la mortalité (réduction de $1 \%$ de la mortalité par gramme de protéines ingérées chaque jour) [31]. Dans une étude prospective, la majoration de l'apport protéique est corrélée à un meilleur pronostic à l'inverse de l'apport énergétique seul [32]. L'apport précoce élevé en protéines $(>1,2 \mathrm{~g} / \mathrm{kg}$ de protéines à j4) a permis de réduire la mortalité [33]. Néanmoins, des essais prospectifs récents montrent des résultats contradictoires. Un travail randomisé comparant $0,8 \mathrm{~g} / \mathrm{kg}$ d'apport en protéines versus $1,2 \mathrm{~g} / \mathrm{kg}$ par jour chez des patients de réanimation a démontré que les patients du groupe à haute teneur en protéines présentaient moins de fatigue et une plus grande épaisseur musculaire aux ultrasons, avec cependant aucune différence en termes de mortalité [32]. Dans une autre étude, un apport protéique élevé au cours de la première semaine a été associé à une plus grande perte musculaire [34]. De plus, sur la base d'une analyse post hoc de l'essai EPANIC, il est suggéré des effets négatifs de l'apport protéique au cours des trois premiers jours d'admission, attribués à une réduction des mécanismes d'autophagie secondaire à l'apport excessif en protéines [35].

Les nombreuses disparités entre les différentes études suggèrent qu'il n'existe pas à ce jour de ratio calories/protéines défini pouvant être appliqué à tous les patients et dans toutes les conditions pathologiques. Ainsi, comme pour l'apport énergétique, nous ne connaissons pas la quantité optimale de protéines en termes pronostiques chez le patient de réanimation. C'est dans ce contexte que le développement de biomarqueurs ou de techniques évaluant la composition corporelle (échographie, scanner) pourrait être une aide à la prise en charge métabolique sous réserve de sa démonstration.

\section{Quelles modalités d'administration du support nutritionnel ?}

Les conséquences potentiellement graves de la dénutrition, en particulier chez les sujets âgés et/ou déjà dénutris à leur admission, justifient la mise en place d'un support nutritionnel. Ainsi, le support métabolique devra être initié chez le patient de réanimation selon différentes modalités d'administration.

Les recommandations de la SFAR-SRLF-SFNEP sont [18]:

- il faut administrer dans les 24 premières heures un support nutritionnel entéral aux patients dénutris ou jugés incapables de s'alimenter suffisamment dans les trois jours après l'admission (accord fort) ;

- il faut utiliser la nutrition entérale (NE) plutôt que la nutrition parentérale (NP), en l'absence de contre-indication formelle (accord fort)

- il faut utiliser la NP en présence de contre-indication formelle de la NE (accord faible). En cas de NP exclusive, il est nécessaire d'administrer des micronutriments selon les recommandations.

\section{Nutrition entérale : avantages et inconvénients}

Lorsque l'alimentation orale est impossible et que le tube digestif est fonctionnel, la NE doit être le support nutritionnel privilégié, ce qui est une pratique standard et maintenant recommandée par de nombreuses sociétés savantes. En effet, par rapport à la NP, la NE offre les avantages suivants : elle est plus physiologique, moins invasive, avec notamment moins de complications infectieuses, préservant la trophicité et la fonction absorptive de la muqueuse intestinale ; enfin, elle est aussi moins coûteuse [36]. Plusieurs méta-analyses confirment la réduction de la morbimortalité avec l'instauration d'une NE précoce $[37,38]$. Une NE instaurée précocement permet de limiter le déficit énergétique, tout en protégeant la trophicité du tube digestif. Dans une étude, les patients avec des vasopresseurs et une alimentation entérale dans les 48 premières heures ont une survie significativement meilleure, comparativement aux patients avec un délai de mise en route du support nutritionnel entéral [39]. Le timing de mise en route de la NE est un facteur essentiel. Il a été montré une diminution significative de la durée totale de séjour et de la mortalité entre une NE débutée dans les 48 heures suivant l'intubation, comparativement à l'absence de NE précoce [40]. Cependant, une étude, menée chez 150 patients sous ventilation mécanique, a montré que la tentative d'atteindre l'objectif calorique avec de la NE dès le premier jour induisait un nombre plus élevé d'infections et prolongeait la durée de séjour hospitalier par rapport à une augmentation progressive des apports caloriques réalisée sur 
cinq jours [41]. Toutefois, dans cette étude, la NE précoce était loin d'atteindre l'objectif calorique prescrit et optimal.

La mise en route optimale de la NE n'est pas toujours aisée. En effet, la NE est fréquemment retardée ou interrompue pour différentes raisons, comme des examens radiologiques ou endoscopiques, un acte chirurgical, des problèmes mécaniques (obstruction ou mauvais positionnement de la sonde nasogastrique, dysfonction de la pompe de nutrition) ou une intolérance digestive (vomissements, diarrhée, distension abdominale), survenant chez $46 \%$ des patients recevant une NE [42]. La présence d'un état de choc non contrôlé et/ou avec des doses d'amines croissantes doit faire retarder la mise en route de l'apport entéral. Dans la série publiée par Reignier et al incluant des patients en état de choc, les auteurs ont mis en évidence un risque accru d'ischémie mésentérique dans le bras entéral versus parentéral, bien qu'il faille noter un objectif calorique excessif dans le groupe entéral [43]. C'est dans ce contexte que des études ont été réalisées avec une nutrition dite trophique, mais les résultats restent à confirmer [28]. L'absence ou l'inadéquation de protocoles de nutrition peut également entraîner un retard d'administration de la NE. Le fait que la cible énergétique soit rarement atteinte chez les patients recevant une NE exclusive entraîne l'apparition progressive de déficits nutritionnels impliqués dans la morbimortalité des patients.

En ce qui concerne la mortalité, les données sont plus contrastées et discutées. En effet, les résultats de métaanalyses ne montrent pas de différence significative entre les deux voies d'administration sur la mortalité [44].

\section{Nutrition parentérale : avantages et inconvénients}

La NP exclusive est indiquée pour les patients qui ne tolèrent pas et/ou qui présentent des contre-indications absolues à la NE. Jusqu'à récemment, il était considéré que la mise en place d'une NP précoce en phase aiguë n'apportait aucun bénéfice du fait des complications métaboliques (hyperglycémie, hypertriglycéridémie), endocriniennes et infectieuses observées [45]. L'atrophie de la muqueuse intestinale, à l'origine du risque de translocation bactérienne, a notamment été incriminée dans l'augmentation de l'incidence des infections et des septicémies sous NP. Cependant, de nombreuses études menées chez l'homme ont montré que la NP exclusive n'induisait pas d'atrophie intestinale, même après un mois de mise au repos complet du tube digestif [46]. Les complications associées à l'utilisation de la NP semblent généralement être plutôt le résultat d'une administration excessive de calories, associée à un contrôle glycémique insuffisant. La NP peut être administrée sans complication si elle est utilisée par une équipe entraînée, avec des apports énergétiques en adéquation avec la cible [47]. La métaanalyse compilant 11 études comparant la NE et la NP exclusive confirme cette hypothèse en montrant un bénéfice de la
NP exclusive en termes de mortalité, et ce, malgré une augmentation du taux de complications infectieuses dans le groupe recevant la NP [48]. Le bénéfice de la NP était encore plus marqué lorsque étaient pris en compte les essais comparant l'utilisation de la NP exclusive précoce à la NE retardée ( $>24$ heures). Les auteurs concluaient que le bénéfice clinique de la NP précoce était supérieur à celui d'une NE retardée. L'étude CALORIES comparant la NP à la NE chez 2400 patients en soins intensifs n'a montré aucune différence dans la mortalité à j90, les infections, les durées de séjour et de ventilation entre les deux voies d'administration [49]. Il est à noter que dans ce travail les apports caloriques et protéiques étaient identiques dans les deux bras d'études ; seule la voie d'administration était différente. Enfin, l'étude NUTRIREA-2 de Reignier et al. n'a pas montré d'impact négatif à la voie parentérale précoce dans une population de patients sous ventilation mécanique invasive avec un support vasomoteur [43].

\section{Nutrition parentérale complémentaire}

Dans l'état actuel des travaux publiés, il semble préférable de ne pas chercher à compenser trop vite et trop rigoureusement le déficit caloricoazoté observé chez un patient intolérant à la NE. Lorsqu'une NP de complément est administrée, les apports caloriques doivent être ajustés au déficit énergétique (évaluable par calorimétrie indirecte) [50]. Dans l'étude TICACOS, l'optimisation des apports caloriques, ajustés à la mesure par calorimétrie indirecte, permettait d'obtenir une balance calorique positive de $2000 \mathrm{kcal}$ à j14 de l'admission, mais sans effet significativement positif sur la mortalité, la durée de séjour ou le taux d'infections [51]. Il a été conclu que la SPN précoce chez les patients à faible risque en soins intensifs ne confère pas d'avantage.

\section{Quelle place pour l'immunonutrition?}

De faibles taux plasmatiques de glutamine sont fréquemment rencontrés et associés à une mortalité accrue. La supplémentation a été envisagée avec l'hypothèse suivante que la glutamine devient un acide aminé essentiel. Les premières études observationnelles avec une supplémentation en glutamine à faible dose ont montré des résultats significatifs en termes de réduction de mortalité et de taux d'infection. Les essais REDOXS et MetaPlus ont montré des impacts négatifs en termes de mortalité $[52,53]$. Dans ces essais, la glutamine a été administrée sans que l'on connaisse les taux de base de glutamine, et l'hyperglutaminémie peut avoir conduit aux résultats négatifs. Dans l'étude REDOXS, les doses de glutamine administrées étaient très importantes ( $30 \mathrm{~g} / \mathrm{j}$ par voie entérale et $0,35 \mathrm{~g} / \mathrm{kg}$ et par jour par voie parentérale), bien au-dessus des doses recommandées 
habituellement. Rodas et al. ont montré qu'un taux élevé de glutamine est associé négativement à la survie [54]. Les méta-analyses récentes ne montrent aucun impact bénéfique de son utilisation [55].

Dans la méta-analyse de Manzanares et al regroupant dix essais randomisés, il n'existe aucun impact sur la mortalité [56]. Par conséquent, aucune recommandation ne peut être faite à ce jour pour un apport systématique en huile de poisson quelle que soit la voie d'administration. Des études futures randomisées sur une population homogène pourraient peut-être modifier ces propos.

\section{Conclusion}

Le support métabolique au cours de l'agression reste un sujet complexe. Les récentes études ont considérablement changé notre compréhension à la fois sur la physiopathologie du métabolisme de l'agressé et sur les différents aspects de sa prise en charge, incluant le monitorage de la réponse métabolique, la détermination des besoins protéinoénergétiques, les modalités d'administration et autres supports. Face à des patients de plus en plus âgés, dénutris ou avec de nombreuses comorbidités, la prise en charge nutritionnelle optimale s'annonce comme un enjeu primordial. Ainsi, en réanimation, la nutrition artificielle doit faire partie intégrante de la prise en charge du patient, au même titre que la ventilation, l'assistance circulatoire, la suppléance rénale et l'antibiothérapie.

Liens d'intérêts : les auteurs déclarent ne pas avoir de lien d'intérêt.

\section{Références}

1. Dhar A, Castillo L, (2011) Insulin resistance in critical illness. Curr Opin Pediatr 23: 269-274

2. Fahy BG, Sheehy AM, Coursin DB, (2009) Glucose control in the intensive care unit. Crit Care Med 37: 1769-1776

3. Virkamaki A, Puhakainen I, Koivisto V, Vuorinen-Markkola H, Yki-Järvinen H, (1992) Mechanisms of hepatic and peripheral insulin resistance during acute infections in humans. J Clin Endocr Met 74: 673-679

4. Weber-Carstens S, Schneider J, Wollersheim T, Assmann A, Bierbrauer J, Marg A, Al Hasani H, Chadt A, Wenzel K, Koch S, Fielitz J, Kleber C, Faust K, Mai K, Spies CD, Luft FC, Boschmann M, Spranger J, Spuler S, (2013) Critical illness myopathy and GLUT4: significance of insulin and muscle contraction. Am J Respir Crit Care Med 187: 387-396

5. Tappy L, Chioléro R, (2007) Substrate utilization in sepsis and multiple organ failure. Crit Care Med 35: S531-S534

6. Gladden LB, (2004) Lactate metabolism: a new paradigm for the third millennium. J Physiol 1: 5-30

7. Marik PE, (2006) Dyslipidemia in critically ill. Crit Care Clin 22: $151-159$
8. Wolfe RR, (1999) Sepsis as a modulator of adaptation to low and high carbohydrate and low and high fat intakes. Eur J Clin Nutr 53: S136-S142

9. Biolo G, (2013) Protein metabolism and requirements. World Rev Nutr Diet 105: 12-20

10. Looijaard WG, Dekker IM, Stapel SN, Girbes AR, Twisk JW, Oudemans-van Straaten HM, Weijs PJ, (2016) Skeletal muscle quality as assessed by CT-derived skeletal muscle density is associated with 6-month mortality in mechanically ventilated critically ill patients. Crit Care 20: 386

11. Cartwright MM, (2004) The metabolic response to stress: a case of complex nutrition support management. Crit Care Nurs Clin North Am 16: 467-487

12. Cynober L, de Bandt JP, (2014) Glutamine in the intensive care unit. Curr Opin Clin Nutr Metab Care 17: 98-104

13. Batch BC, Hyland K, Svetkey LP, (2014) Branch chain amino acids: biomarkers of health and disease. Curr Opin Clin Nutr Metab Care 17: 86-89

14. Mickiewicz B, Duggan GE, Winston BW, Doig C, Kubes P, Vogel HJ; Alberta Sepsis Network, (2014) Metabolic profiling of serum samples by $1 \mathrm{H}$ nuclear magnetic resonance spectroscopy as a potential diagnostic approach for septic shock. Crit Care Med 42: 1140-1149

15. Chioléro R, Revelly JP, Tappy L, (1997) Energy metabolism in sepsis and injury. Nutrition 13: 45S-51S

16. Plank LD, Hill GL, (2003) Energy balance in critical illness. Proc Nutr Soc 62: 545-552

17. Haugen HA, Chan LN, Li F, (2007) Indirect calorimetry: a practical guide for clinicians. Nutr Clin Pract 22: 377-388

18. Lefrant JY, Hurel D, Cano NJ, Ichai C, Preiser JC, Tamion F; Société française d'anesthésie et de réanimation; Société de réanimation de langue française; Société francophone nutrition clinique et métabolique, (2014) Recommandations formalisées d'experts. Nutrition artificielle en réanimation. Ann Fr Anesth Reanim 33: 202-218

19. Dvir D, Cohen J, Singer P, (2006) Computerized energy balance and complications in critically ill patients: an observational study. Clin Nutr 25: 37-44

20. Villet S, Chiolero RL, Bollmann MD, Revelly JP, Cayeux RNMC, Delarue J, Berger MM, (2005) Negative impact of hypocaloric feeding and energy balance on clinical outcome in ICU patients. Clin Nutr 24: 502-509

21. Alberda C, Gramlich L, Jones N, Jeejeebhoy K, Day AG, Dhaliwal R, Heyland DK, (2009) The relationship between nutritional intake and clinical outcome in critically ill patients. Intensive Care Med 35: 1728-1737

22. Casaer MP, Mesotten D, Hermans G, Wouters PJ, Schetz M, Meyfroidt G, Van Cromphaut S, Ingels C, Meersseman P, Muller J, Vlasselaers D, Debaveye Y, Desmet L, Dubois J, Van Assche A, Vanderheyden S, Wilmer A, Van den Berghe G, (2011) Early versus late parenteral nutrition in critically ill adults. $\mathrm{N}$ Engl $\mathrm{J}$ Med 365: 506-517

23. Arabi YM, Aldawood AS, Haddad SH, Al-Dorzi HM, Tamin HM, Jones G, Mehta S, Al-McIntyre L, Solaiman O, Sakkijha MH, Sadat M, Afesh L Permit Trial Group, (2015) Permissive underfeeding or standard enteral feeding in critically ill adults. N Engl J Med 372: 2398-2408

24. Rugeles S, Villarraga-Angulo LG, Ariza-Gutierrez A, ChaverraKornerup S, Lasalvia P, Rosselli D, (2016) High-protein hypocaloric versus normocaloric enteral nutrition in critically ill patients: a randomized clinical trial. J Crit Care 35: 110-114

25. Petros S, Horbach M, Seidel F, Weidhase L, (2016) Hypocaloric versus normocaloric nutrition in critically ill patients: a prospective randomized pilot trial. JPEN J Parenter Enteral Nutr 40: 242-249 
26. Choi EY, Park DA, Park J, (2015) Calorie intake of enteral nutrition and clinical outcomes in acutely critically ill patients: a metaanalysis of randomized controlled trials. JPEN J Parenter Enteral Nutr 39: 291-300

27. Marik PE, Hooper MH, (2016) Normocaloric versus hypocaloric feeding on the outcomes of ICU patients: a systematic review and meta-analysis. Intensive Care Med 42: 316-323

28. Nicolo M, Heyland DK, Chittams J, Sammarco T, Compher C, (2016) Clinical outcomes related to protein delivery in a critically ill population: a multicenter, multinational observation study. JPEN J Parenter Enteral Nutr 40: 45-51

29. Ishibashi N, Plank LD, Sando K, Hill GL, (1998) Optimal protein requirements during the first 2 weeks after onset of critical illness. Crit Care Med 26: 1529-1535

30. Koretz RL, Avenell A, Lipman TO, Braunschweig CL, Milne AC, (2007) Does enteral nutrition affect clinical outcome? A systematic review of the randomized trials. Am J Gastroenterol 102: 412-429

31. Mehta NM, Bechard LJ, Zurakowski D, Duggan CP, Heyland DK, (2015) Adequate enteral protein intake is inversely associated with 60-d mortality in critically ill children: a multicenter, prospective, cohort study. Am J Clin Nutr 102: 199-206

32. Ferrie S, Allman-Farinelli M, Daley M, Smith K, (2016) Protein requirements in the critically ill: a randomized controlled trial using parenteral nutrition. JPEN J Parenter Enteral Nutr 40: 795-805

33. Weijs PJ, Looijaard WG, Beishuizen A, Girbes AR, Oudemansvan Straaten HM, (2014) Early high protein intake is associated with low mortality and energy overfeeding with high mortality in nonseptic mechanically ventilated critically ill patients. Crit Care 18: 701

34. Puthucheary ZA, Rawal J, McPhail M, Connolly B, Ratnayake G, Chan P, Hopkinson NS, Phadke R, Dew T, Sidhu PS, Velloso C, Seymour J, Agley CC, Selby A, Limb M, Edwards LM, Smith K, Rowlerson A, Rennie MJ, Moxham J, Harridge SD, Hart N, Montgomery HE, (2013) Acute skeletal muscle wasting in critical illness. JAMA 310: 1591-1600

35. Casaer MP, Wilmer A, Hermans G, Wouters PJ, Mesotten D, Van den Berghe G, (2013) Role of disease and macronutrient dose in the randomized controlled EPaNIC trial: a post hoc analysis. Am J Respir Crit Care Med 187: 247-255

36. Marik PE, (2001) Early enteral nutrition in acutely ill patients: a systematic review. Crit Care Med 29: 2264-2270

37. Heyland DK, Dhaliwal R, Drover JW, Gramlich L, Dodek P; Canadian Critical Care Clinical Practice Guidelines Committee, (2003) Canadian clinical practice guidelines for nutrition support in mechanically ventilated, critically ill adult patients. J Parenter Enteral Nutr 27: $355 \mathrm{e} 73$

38. Doig GS, Heighes PT, Simpson F, Sweetman EA, Davies AR, (2009) Early enteral nutrition, provided within $24 \mathrm{~h}$ of injury or intensive care unit admission, significantly reduces mortality in critically ill patients: a metaanalysis of randomised controlled trials. Intensive Care Med 35: 2018-2027

39. Khalid I, Doshi P, DiGiovine B, (2010) Early enteral nutrition and outcomes of critically ill patients treated with vasopressors and mechanical ventilation. Am J Crit Care 19: 261-268

40. Ibrahim EH, Mehringer L, Prentice D, Sherman G, Schaiff R, Fraser V, Kollef MH, (2002) Early versus late enteral feeding of mechanically ventilated patients: results of a clinical trial. JPEN J Parenter Enteral Nutr 26: 174-178

41. Mentec H, Dupont H, Bocchetti M, Cani P, Ponche F, Bleichner $\mathrm{G}$, (2001), Upper digestive intolerance during enteral nutrition in critically ill patients: frequency, risk factors, and complications. Crit Care Med 29: 1955-1961

42. McClave SA, Sexton LK, Spain DA, Adams JL, Owens NA, Sullins MB, Blandford BS, Snider HL, (1999) Enteral tube feeding in the intensive care unit: factors impeding adequate delivery. Crit Care Med 27: 1252-1256

43. Reignier J, Boisramé-Helms J, Brisard L, Lascarrou JB, Ait Hssain A, Anguel N, Argaud L, Asehnoune K, Asfar P, Bellec F, Botoc V, Bretagnol A, Bui HN, Canet E, Da Silva D, Darmon M, Das V, Devaquet J, Djibre M, Ganster F, Garrouste-Orgeas M, Gaudry S, Gontier O, Guérin C, Guidet B, Guitton C, Herbrecht JE, Lacherade JC, Letocart P, Martino F, Maxime V, Mercier E, Mira JP, Nseir S, Piton G, Quenot JP, Richecoeur J, Rigaud JP, Robert R, Rolin N, Schwebel C, Sirodot M, Tinturier F, Thévenin D, Giraudeau B, Le Gouge A; NUTRIREA-2 Trial Investigators; Clinical Research in Intensive Care and Sepsis (CRICS) group, (2018) Enteral versus parenteral early nutrition in ventilated adults with shock: a randomised, controlled, multicentre, open-label, parallelgroup study (NUTRIREA-2). Lancet 391: 133-143

44. Elke G, van Zanten AR, Lemieux M, McCall M, Jeejeebhoy KN, Kott M, Jiang X, Day AG, Heyland DK, (2016) Enteral versus parenteral nutrition in critically ill patients: an updated systematic review and meta-analysis of randomized controlled trials. Crit Care 20: 117

45. Marik PE, Pinsky M, (2003) Death by parenteral nutrition. Intensive Care Med 29: 867-869

46. Jeejeebhoy KN, (2001) Total parenteral nutrition: potion or poison? Am J Clin Nutr 74: 160-163

47. Nardo P, Dupertuis YM, Jetzer J, Kossovsky MP, Darmon P, Pichard C, (2008) Clinical relevance of parenteral nutrition prescription and administration in 200 hospitalized patients: a quality control study. Clin Nutr 27: 858-864

48. Simpson F, Doig GS, (2005) Parenteral versus enteral nutrition in the critically ill patient: a meta-analysis of trials using the intention to treat principle. Intensive Care Med 31: 12-23

49. Harvey SE, Parrot F, Harrison DA, Bear DE, Segaran E, Beale R, Bellingan G, Leonard R, Mythen MG, Rowan KM; CALORIES Trial Investigators, (2014) Trial of the route of early nutritional support in critically ill adults. N Engl J Med 371: 1673-1684

50. Heidegger CP, Berger MM, Graf S, Zingg W, Darmon P, Costanza MC, Thibault R, Pichard C, (2012) Optimization of energy provision with supplemental parenteral nutrition (SPN) improves the clinical outcome of critically ill patients: a randomized controlled clinical trial. Lancet 381: 385-393

51. Singer P, Anbar R, Cohen J, Shapiro H, Shalita-Chesner M, Lev S, Grozovski E, Theilla M, Frishman S, Madar Z, (2011) The tight calorie control study (TICACOS): a prospective, randomized, controlled pilot study of nutritional support in critically ill patients. Intensive Care Med 37: 601-609

52. Heyland D, Muscedere J, Wischmeyer PE, Cook D, Jones G, Albert M, Elke G, Berger MM, Day AG; Canadian Critical Care Trials Group, (2013) A randomized trial of glutamine and antioxidants in critically ill patients. N Engl J Med 368: 1489-1497

53. van Zanten AR, Sztark F, Kaisers UX, Zielmann S, Felbinger TW, Sablotzki AR, De Waele JJ, Timsit JF, Honing ML, Keh D, Vincent JL, Zazzo JF, Fijn HB, Petit L, Preiser JC, van Horssen PJ, Hofman Z, (2014) High-protein enteral nutrition enriched with immune-modulating nutrients versus standard high-protein enteral nutrition and nosocomial infections in the ICU: a randomized clinical trial. JAMA 312: 514-524

54. Rodas PC, Rooyackers O, Hebert C, Norberg Å, Wernerman J, (2012) Glutamine and glutathione at ICU admission in relation to outcome. Clin Sci (Lond) 122: 591-597

55. Wischmeyer PE, Dhaliwal R, McCall M, Ziegler TR, Heyland DK, (2014) Parenteral glutamine supplementation in critical illness: a systematic review. Crit Care 18: R76

56. Manzanares W, Langlois PL, Dhaliwal R, Lemieux M, Heyland DK, (2015) Intravenous fish oil lipid emulsions in critically ill patients: an updated systematic review and metaanalysis. Crit Care 19: 167 